profound effect on the continuous spectrum of an $A$ star, as shown by the curve computed by Panne. koek for a temperature $T_{0}=8,480^{\circ}$, and it must have a marked influence on the ionization as well as on the excitation of those elements the ionization potentials of which lie in the region of 12 volts. Research in recent months has led the authors independently to the conclusion that this physical dilution of radiation-as contrasted with the geometrical dilution observed in shells-plays a very important part in a number of problems, and various instances of this are given. A wide variety of unexplained observations are capable of interpretation along the general lines suggested by the authors, details of which are to be published in the Astrophysical Journal. Departures from thermodynamic equilibrium are now being emphasized by recent developments in stellar spectroscopy, in contrast to the long and successful period characterized by the use of thermodynamic equilibrium.

\section{Infra-red Baking}

A RECEnT article by H. Seymour (The Electrician, August 21) surveys the present state of the technique of infra-red baking of industrial finishes. The article makes brief reference to early development and mentions that although first patented some seven years ago, radiant heating received little attention. until adopted by the Ford Motor Co. It is emphasized that the rate of temperature rise depends primarily on the difference between the energy gain of the stock by absorption of rediation, and the loss of heat by re-radiation and convection; and that the temperature of the stock and its surroundings should be of the same order of magnitude. Depending on the linear absorption coefficient and absorptivity of the film, baking or heating may occur from the inside outwards, the outside inwards, or uniformly throughout the film.

A description is included of the various types of radiant heating lamps, and of their early development ; reflectors and their surfaces; and the present trend of design. Reflector surface fouling by dust and fumes receives special mention, while reference is made to a new type of unit with a sealed lens which obviates cleansing, reduces the fire hazard, and avoids reflector surface damage. An intensity survey is included which discusses the possibilities of obtaining uniform energy intensities over surfaces normal to the lamps. The results of recent research into the infra-red absorption characteristics of various paint pigments ground in similar vehicles are given. The conditions for maximum utilization of electrical energy are examined and it is stated that this obtains when the air temperature is higher than the stock temperature. Vapour removal, performance ratio, maximum heat densities and oven arrangements are discussed, and the view is expressed that future practice will utilize radiant heat lamps augmented by auxiliary heaters.

\section{Soldering Electrical Joints}

Av article in the Electrical Review of August 28 describes the technique and selection of suitable materials. The process of soldering may be defined as the uniting of metals by means of an alloy melting at a lower temperature than their fusing points, as distinct from welding in a state of fusion. The solder alloys with the surface layers of the parts to be joined and the process is not simply one of adhesion. There are two broad classes of soldering, namely, soft soldering in which tin-lead alloys are mainly used and hard soldering in which alloys of copper-zinc (brazing) or of silver-copper-zinc (silver soldering) are employed. Soft soldering is used very extensively in electrical work and silver soldering to some considerable extent, brazing is rarely used. The electrical conductivity of soft and hard solders is considerably less than that of copper, varying with composition between approximately 9 per cent and 13 per cent for soft solders and 20 per cent and 40 per cent for silver solders.

Some or all of the following points must be considered in the selection of soldering processes for joints in current-carrying conductors. (1) Thorough cleansing and adequate heating, but not overheating. (2) As little solder as possible should be used, to obtain low electrical resistance and high mechanical strength. (3) Solder must not weaken at normal working temperatures. (4) Due to high centrifugal forces encountered in many electrical joints (for example, soldered commutator connexions), solder must be both strong and ductile. (5) Fluxes of a corrosive nature must be avoided, and flux residue easily removable. (6) Solders used for joining copper in the manufacture of cables must be strong and ductile to withstand the subsequent drawing operations; silver solders are generally employed. The article gives tables of solder compositions, properties and characteristics.

\section{Health of the Swedish Army}

According to the August issue of the AngloSwedish Review, General Ivar Holmquist, Commanderin-Chief of the Swedish Army, has recently declared that, without going into technical details, he was sure that the Swedish soldier was one of the best in the world. What he lacked was only battle experience, but he was at least partly likely to make up for this by his excellent physique. Moreover, the nerves of the soldiers were in good order, they did not suffer from war weariness, the material was in first-class condition and had not been worn out by war, nor had the élite troops been decimated. Although it was deplorable that so much of the country's labour and resources had to be used for military purposes, the effort was not altogether wasted. For the general health of the nation it was important that the young men of twenty should receive one year's excellent physical training in the open air. During a recent tour of inspection among the mountains of Lapland, he was pleased to see with what vitality and enthusiasm these young men carried out a march of 13 miles in alpine terrain rising from 1,000 to 3,000 feet and then delivered an attack down a mountain slope carrying the heavy equipment of machine-guns and trench mortars which had been conveyed on sledges during the march.

\section{Public Health in Peru}

IN a recent article (Bol. Of. San. Panamericana, 21, 540 ; 1942 ) Dr. Constantino J. Carvallo, the Minister of Public Health, Labour and Social Welfare, gives a description of public health in Peru from December 1939 to July 1941. The chief health problems of this country are malaria, tuberculosis, children's diseases, venereal diseases and other contagious disorders. Health campaigns which have already begun include surveys, health examinations, vaccination, establishment of clinics and dispensaries, early treat- 